\title{
Aberration-Corrected STEM Study of Shape Controlled Metallic Core-Shell Nanoparticles for Catalytic Applications
}

\author{
Jinguo Wang ${ }^{1}$, Ning $\mathrm{Lu}^{1}$, Younan $\mathrm{Xia}^{2,3}$ and Moon J. Kim ${ }^{1}$ \\ 1. Department of Materials Science and Engineering, The University of Texas at Dallas, Richardson, \\ Texas. \\ 2. The Wallace H. Coulter Department of Biomedical Engineering, Georgia Institute of Technology and \\ Emory University, Atlanta, Georgia. \\ 3. School of Chemical Biochemistry, Georgia Institute of Technology, Atlanta, Georgia.
}

Thanks for the rapid development of nanotechnology over the past few decades, nanoparticles (NPs) containing noble metals as active catalytic components, can now be routinely synthesized with welldefined sizes, shapes, crystal facets, structure and composition. In the catalysis area, one important challenge is to development a highly active and selective, robust, low-cost and environmentally benign catalytic system, which could be addressed by developing catalytic systems with core-shell structural features. Core-shell nanoparticles could offer many advantages which allow the specific surface of the core be coated with shell elements, thus resulting in superior catalytic performance of such a layer/shell. In general, the activity and durability of a catalyst can be improved by engineering the size, shape, structure, and composition of the nanoparticles involved. Our studies show that by controlling the size, shape, shell thickness, and composition, Pt-based bimetallic core-shell nanoparticles could demonstrate remarkable catalytic performance [1].

In this report, we summarize our recent studies on the size, shape, atomic structures and composition distribution of the Pt or Pt alloy shell layer in various core shell nanoparticles by using aberration (Cs) corrected high angle angular dark field (HAADF) - scanning transmission electron microscopy (STEM), and energy dispersive X-ray spectroscopy (EDS). We also demonstrate that these core-shell nanoparticles have superior catalysis performance. Core-shell nanoparticles were synthesized by sitespecific growth on the cores with different shapes [2-4]. TEM, HAADF-STEM, and EDS mapping were performed in a JEOL ARM200F with a STEM Cs corrector operated at $200 \mathrm{kV}$. Electrochemical measurement was also performed to measure the specific and mass activities of the core-shell nanoparticles.

Atomic resolution Cs-corrected HAADF imaging combined with EDS line scanning has been proven to be an effective tool to identify the shape, structure and composition of core-shell nanoparticles at atomic level [1]. Figure 1 shows the results of EDS line scanning identifies Pt atomic layers in Pd-Pt core-shell octahedron. Figure 1a is a HAADF image of a Pd-Pt core-shell octahedron, Figure 1(b) is a local enlarged HAADF -STEM image, where the red arrow indicates the direction of EDS line scanning. The corresponding line profile of EDS scanning (Figure 1c) confirms the structure of Pt shell/Pd core. The Pd-Pt core shell octahedra exhibited at least 4-fold enhancement in specific activity toward the oxygen reduction reaction (ORR) when compared to a commercial $\mathrm{Pt} / \mathrm{C}$ based upon $3.2 \mathrm{~nm} \mathrm{Pt}$ particles [3]. Figure 2 shows the HAADF-STEM image of a Pt-Ni alloy octahedral nanocrystal and (d) EDS line scan showing Pt and Ni distributions along the arrow marked in (c). For a catalyst based on Pt-Ni octahedra of $9 \mathrm{~nm}$ in edge length, it has demonstrated an ORR mass activity of $2.67 \mathrm{~A} \mathrm{mg}_{\mathrm{Pt}}^{-1}$ at $0.9 \mathrm{~V}$, representing an 11-fold improvement over a state-of-the-art commercial $\mathrm{Pt} / \mathrm{C}$ catalyst $\left(0.24 \mathrm{~A} \mathrm{mg}_{\mathrm{Pt}}{ }^{-1}\right)$. Core-shell nanoparticles have emerged as valuable and versatile nanomaterials for catalysis because of 
their additive or complementarily enhanced properties compared to their one-component counterparts [5].

\section{References:}

[1] N. Lu et al, The Journal of Physical Chemistry C 118 (2014), p.28876.

[2] G. Niu et al, Nano Letters 16(6) (2016), p.3850.

[3] J Park et al, ACS nano 9(3) (2015), p.2635.

[4] S. Choi et al, ACS Nano 8(10) (2014), p.10363.

[5] This work was supported in part by Louis Beecherl, Jr. endowment funds. The synthesis work was supported in part by a grant from the NSF (DMR-1215034) and start-up funds from Georgia Institute of Technology.
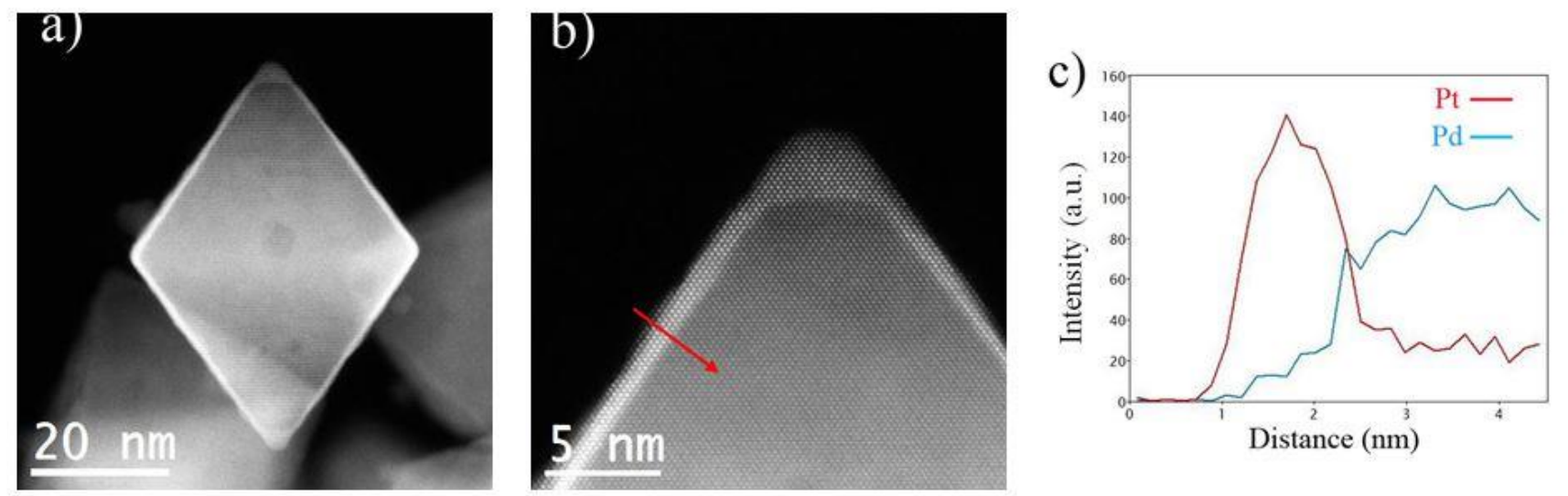

Figure 1. a) a HAADF image of a Pd-Pt core-shell octahedron, (b) a local enlarged HAADF -STEM image, where the red arrow indicates the direction of EDS line scanning. The corresponding line profile of EDS scanning (Figure 1c) confirms the structure of Pt shell/Pd core.
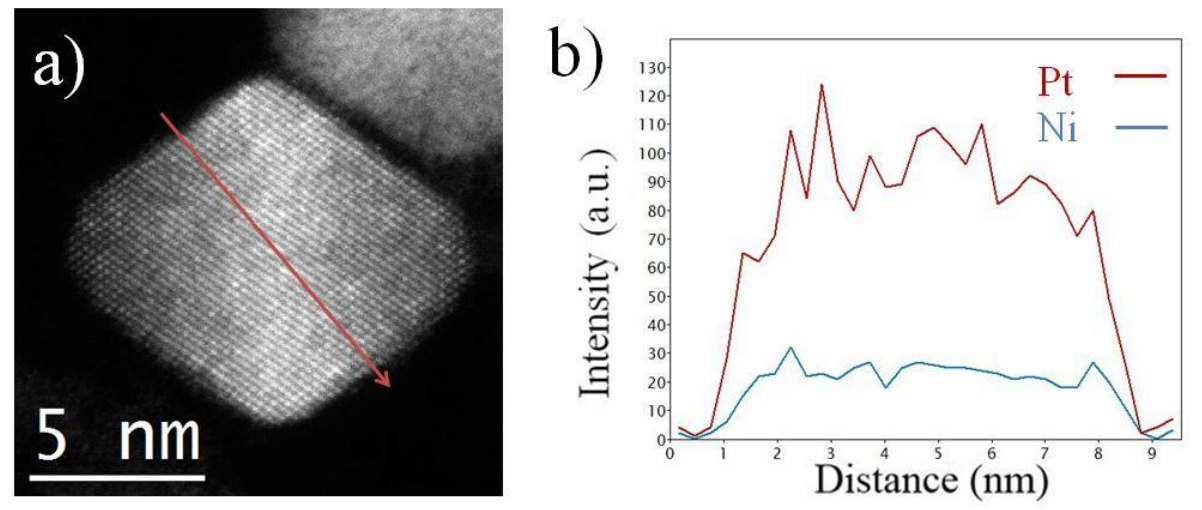

Figure 2. (a) HAADF-STEM image of a Pt-Ni alloy octahedral nanocrystal and (d) EDS line scan showing Pt and Ni distributions along the arrow marked in (c). 